\title{
BMJ Global Health Effect of the competency-based Helping Mothers Survive Bleeding after Birth (HMS BAB) training on maternal morbidity: a cluster-randomised trial in 20 districts in Tanzania
}

\author{
Fadhlun Alwy Al-beity, ${ }^{\oplus 1,2}$ Andrea Pembe, ${ }^{2}$ Atsumi Hirose, ${ }^{1}$ Jessica Morris, ${ }^{3}$ \\ Sebalda Leshabari, ${ }^{4}$ Gaetano Marrone, ${ }^{1}$ Claudia Hanson ${ }^{\circ 1,5}$
}

To cite: Alwy Al-beity F, Pembe A, Hirose A, et al. Effect of the competencybased Helping Mothers Survive Bleeding after Birth (HMS BAB) training on maternal morbidity: a clusterrandomised trial in 20 districts in Tanzania. BMJ Glob Health 2019;4:e001214. doi:10.1136/ bmjgh-2018-001214

Handling editor Stephanie M Topp

- Additional material is published online only. To view please visit the journal online (http://dx.doi.org/10.1136/ bmjgh-2018-001214).

Received 11 October 2018 Revised 5 February 2019 Accepted 10 February 2019

Check for updates

C Author(s) (or their employer(s)) 2019. Re-use permitted under CC BY-NC. No commercial re-use. See rights and permissions. Published by BMJ.

For numbered affiliations see end of article.

Correspondence to Dr Fadhlun Alwy Al-beity; fadhlun.alwy.al-beity@ki.se

\begin{abstract}
Background Training health providers is an important strategy to improve health. We conducted a clusterrandomised two-arm trial in Tanzania to assess the effect of a 1-day competency-based training 'Helping Mothers Survive Bleeding after Birth (HMS BAB)' followed by eight weekly drills on postpartum haemorrhage (PPH)-related morbidity and mortality.
\end{abstract}

Methods Twenty districts in four purposefully selected regions in Tanzania included 61 facilities. The districts were randomly allocated using matched pairs to ensure similarity in terms of district health services in intervention and comparison districts. In the 10 intervention districts 331 health providers received the HMS BAB training. The other half continued with standard practices. We used the WHO's near miss tool to collect information on severe morbidity (near misses) of all women admitted to study facilities. We performed interrupted time series analysis to estimate differences in the change of near miss per delivery rate and case fatality rates. We also assessed implementation of evidence-based preventive and basic management practices for PPH as secondary outcomes. Results We included 120533 facility deliveries, 6503 near misses and 202 maternal deaths in study districts during study period (November 2014 to January 2017). A significant reduction of PPH near misses was found among women who suffered PPH in the intervention district compared with comparison districts (difference-indifferences of slopes $-5.3,95 \% \mathrm{Cl}-7.8$ to $-2.7, \mathrm{p}<0.001$ ) from a baseline PPH-related near miss rate of $71 \%(95 \%$ $\mathrm{Cl} 60 \%$ to $80 \%$ ). There was a significant decrease in the long-term PPH near miss case fatality (differencein-differences of slopes -4 to 0$)(95 \% \mathrm{Cl}-6.5$ to -1.5 , $p<0.01)$ in intervention compared with the comparison districts. The intervention had a positive effect on the proportion of PPH cases treated with intravenous oxytocin (difference-in-differences of slopes $5.2,95 \% \mathrm{Cl} 1.4$ to 8.9 ) $(\mathrm{p}<0.01)$.

Conclusion The positive effect of the training intervention on PPH morbidity and case fatality suggests that the training addresses important deficits in knowledge and skills.

Trial registration number PACTR201604001582128.

\section{Key questions}

What is already known?

- The Helping Mothers Survive Bleeding after Birth (HMS BAB) training is implemented in varying degrees in a number of low/middle-income countries and studies suggest an improvement in knowledge, skills and confidence to manage postpartum haemorrhage (PPH).

- Evidence is missing on whether the training results in improvements in maternal health outcomes (near misses and mortality).

What are the new findings?

- We report small but significant improvements in immediate and long-term reduction of PPH near misses and PPH case fatality rates in intervention compared with comparison districts suggesting that the training makes an important impact on morbidity and mortality reductions.

- Overall case fatality rates were still high as access to advanced life-saving interventions is very limited.

What do the new findings imply?

- The HMS BAB training, although effective, requires additional support to ensure necessary drugs, equipment and supplies are available to bring sustained positive outcomes.

\section{BACKGROUND}

Despite efforts to address maternal health challenges, most countries with a high burden of poor maternal health outcomes did not meet Millennium Development Goal 5 targets by 2015-Tanzania is no exception. Even with renewed efforts to reach targets set in the Sustainable Development Goals by 2030, maternal mortality remains a global challenge, especially in low/middle-income countries. In 2015 alone, approximately 300000 women died of pregnancy and childbearing-related 
causes, a quarter of which were caused by postpartum haemorrhage (PPH). ${ }^{1}$ In Tanzania, maternal mortality is estimated at 398 deaths per 100000 live births with $25 \%$ of all maternal deaths due to haemorrhage. ${ }^{12}$

Giving birth in a facility, attended by a skilled health provider and within a functioning health system have been identified as critical components for reducing high mortality rates. ${ }^{3}$ There has been a corresponding increase in facility deliveries worldwide. ${ }^{4}$ However, a few country analyses have indicated that despite increases in facility births, reductions in mortality rates have remained insufficient. ${ }^{4-6}$ In response, there is an increased focus on improving the quality of care in facilities, especially in low/middle-income countries ${ }^{7-10}$ and to address the low competency levels among health providers in obstetrics including preventing and managing $\mathrm{PPH} .{ }^{11-15}$

In-service training has long been used to improve health workers' competences with varying degrees of success. More recent advances in educational research have led to the conceptualisation of competency-based training supported by simulations to make learning more effective. ${ }^{16}$ One such training is a 1-day competency-based Helping Mothers Survive Bleeding after Birth (HMS $\mathrm{BAB}$ ) programme using a low-fidelity simulator-the Mama Natalie. ${ }^{18}$

Evidence suggests that the HMS BAB training conducted in low/middle-income countries improves providers' knowledge and skills competency on $\mathrm{PPH}$ prevention. ${ }^{13} 19-21$ While the training has been taken to scale in several countries ${ }^{21} 22$ evidence is missing on whether the training does in fact improve maternal outcomes. To provide data on the effect of the training on maternal outcomes, we conducted two trials in the two countries of Uganda and Tanzania. Here we report on the cluster-randomised trial evaluating the effect of the HMS BAB training on morbidity and case fatality rates in Tanzania.

\section{METHODS \\ Study design}

This cluster-randomised trial including 20 clusters (districts) was conducted to assess the effect of the HMS $\mathrm{BAB}$ training on changes in rates of severe maternal morbidity-defined as maternal near miss, among mothers experiencing PPH in Tanzania. Clusters (districts) were used for the level of randomisation because trainings bring effects through prevention, management and referral, beyond the individual care for women. ${ }^{23}$ The HMS BAB training was provided to 31 facilities in 10 intervention clusters while comparison clusters continued with standard care. A cluster was an administrative health district. Details are presented in the trial protocol paper. ${ }^{23}$

\section{Study setting}

The study was done in four regions of Tanzania: Simiyu and Mwanza (Lake zone) and Lindi and Mtwara
(Southern zone). The regions were purposefully selected by the Ministry of Health, Community Development, Gender, Elderly and Children. The proportion of women delivering in a health facility according to the latest Demographic and Household Survey was 32\% (Simiyu), $53 \%$ (Mwanza), $80 \%$ (Lindi) and $81 \%$ (Mtwara). ${ }^{24}$ Basic emergency obstetric and neonatal care signal functions are not consistently implemented in hospitals and health centres throughout the country, but all hospitals and selected health centres do provide caesarean section services. ${ }^{25-27}$ Eighty-eight per cent of facility births are conducted by various nurse and nurse-midwife cadres or medical assistants and $12 \%$ by clinicians (clinical officers, assistant medical officers and medical officers).

\section{Study sites and participants}

Inclusion criteria for a district (cluster) were having a district hospital and two to three large health centres where at least 3000 deliveries are attended per year, and no other scheduled capacity building for obstetric care. Overall, we included 20 districts with 61 health facilities. We included all women attending the selected facilities for delivery care or obstetric complications from July 2015 to November 2016. All providers working in selected maternity wards and present during the training were enrolled as secondary participants in the trial.

\section{Intervention}

The intervention, the HMS BAB training, was developed by Jhpiego and Laerdal Global Health and includes prevention and basic care for PPH. The basic training which we evaluated has recently been complemented by an advanced training module. ${ }^{28}$ The curriculum of the basic HMS BAB training is described in Box 1. The content is aligned to what is taught in preservice trainings, and aims to reinforce and refresh skills rather than introduce new knowledge and skills. For each facility,

Box 1 Helping Mothers Survive Bleeding after Birth (HMS BAB) competency-based training

\section{The HMS BAB intervention}

- A competency-based 1-day training using a low-fidelity birthing simulator: Mama Natalie, https://www.laerdal.com/products/ simulation-training/obstetrics-paediatrics/mamanatalie/

- The curriculum includes basic delivery care and active management of the third stage of labour (AMTSL), a step-by-step assessment of the mother's condition and blood loss and basic management of postpartum haemorrhage (PPH) including application of intravenous oxytocin, uterine massage and recognition of when to refer and referral preparedness.

- In-facility (different from centrally organised training where a few providers attend from each facility) in-service training where al maternity providers within the facility are trained.

- Uses 'low dose, high frequency' teaching methodology where the initial 1-day training is supplemented by eight weekly sessions to practise scenario-based drills that build on learnt competencies. These sessions are supported by a pair of facility-based peer practice coordinators. 


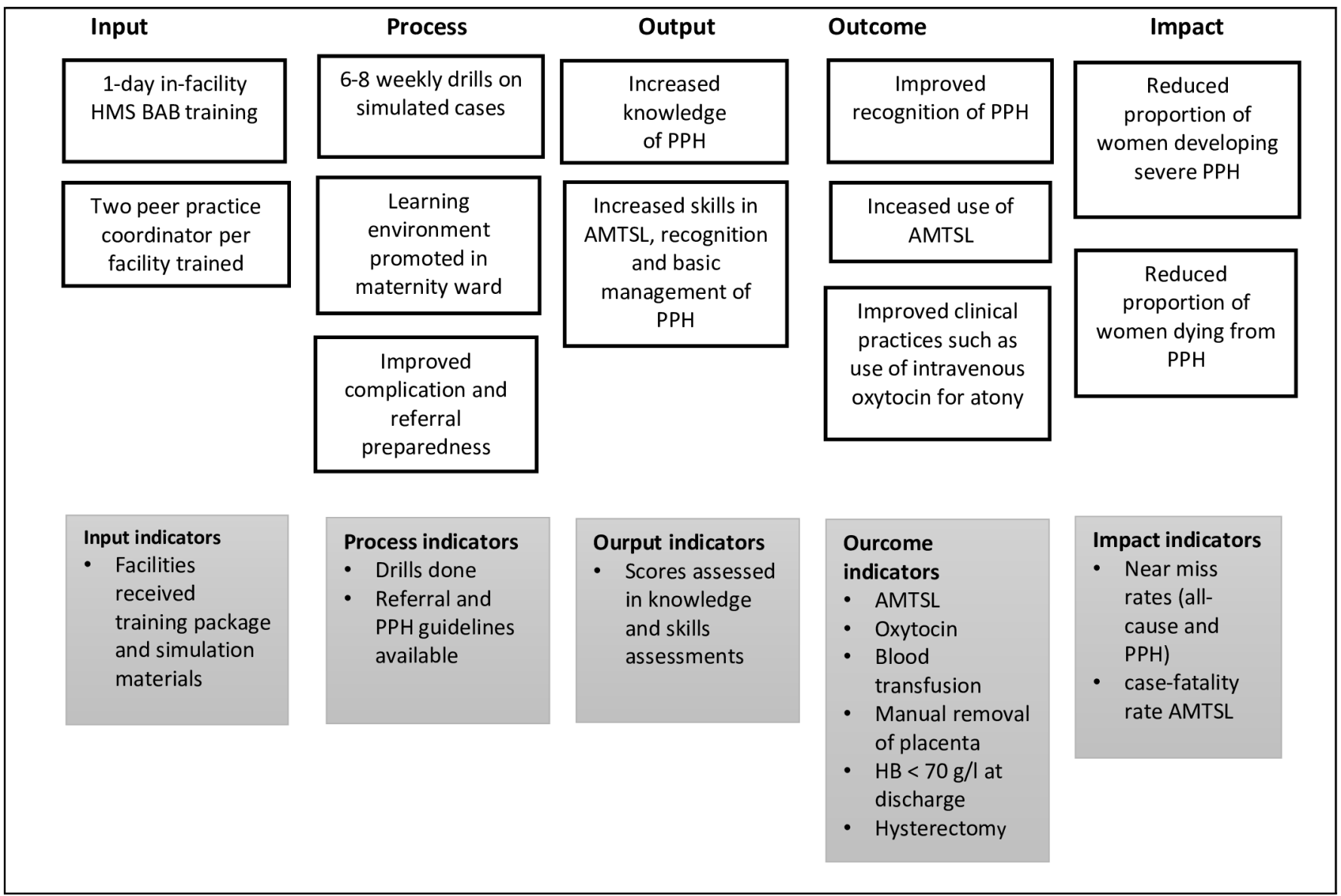

Figure 1 Theory of cChange. AMTSL, active management of the third stage of labour; HMS BAB, Helping Mothers Survive Bleeding after Birth; PPH, postpartum haemorrhage.

training consisted of an initial 1-day training, followed by weekly drill sessions for $6-8$ weeks. These short but frequent training sessions aim to build on learnt skills and enhance skills competency. These 'low dose, high frequency' learning sessions are facilitated by a pair of peer practice coordinators in each facility. Facilities in comparison districts continued with standard practice and received the $\mathrm{HMS} \mathrm{BAB}$ training at the end of the trial (November 2016).

Our theory of change is depicted in figure 1 . We postulate that the training intervention and the frequent drills will improve providers' knowledge and skills. The training should also improve emergency preparedness and the preparation of guidelines for referral at facilities. Improved knowledge and skills and emergency preparedness should lead to consistent implemented preventive practices of active management of the third stage of labour (AMTSL), enhanced recognition of $\mathrm{PPH}$ and early intervention for $\mathrm{PPH}$ such as application of oxytocin. The drills should also stimulate a better learning environment within the facility even when new providers are posted and ultimately will have a sustained effect on skills. Hence, facilities receiving the HMS BAB training will have reduced $\mathrm{PPH}$ case fatality and $\mathrm{PPH}$ near misses.

\section{Implementation}

The implementation followed a stepwise approach with three main steps (figure 2). Step 1 where Jhpiego-trained 'master trainers' facilitated the training of 12 district trainers in a central training. Step 2, the district trainers were accompanied by Jhpiego master trainers to intervention districts. The district trainers were paired, each pair conducted their first in-facility training under the supervision of Jhpiego master trainers to ensure their adherence to training standards. Each pair spent 1.5 days per facility conducting the training. Day 1 was for the HMS training to all providers and day 2 was spent in coaching two local providers to become facility peer practice coordinators. Providers who excelled in the skills training, were willing to lead others and had good communication skills were selected to be peer practice coordinators and received this half-day training and coaching. After this, they were expected to facilitate the six to eight weekly drills using specific scenarios to augment the learnt skills (step 3). A drill session is expected to last approximately 30-40 min.

The in-facility training (step 2) was done between 15 January and 3 February 2015 and 331 providers in 31 health facilities in the 10 randomly selected districts were trained. Facility and labour ward administrators were informed about the date of training a week in 


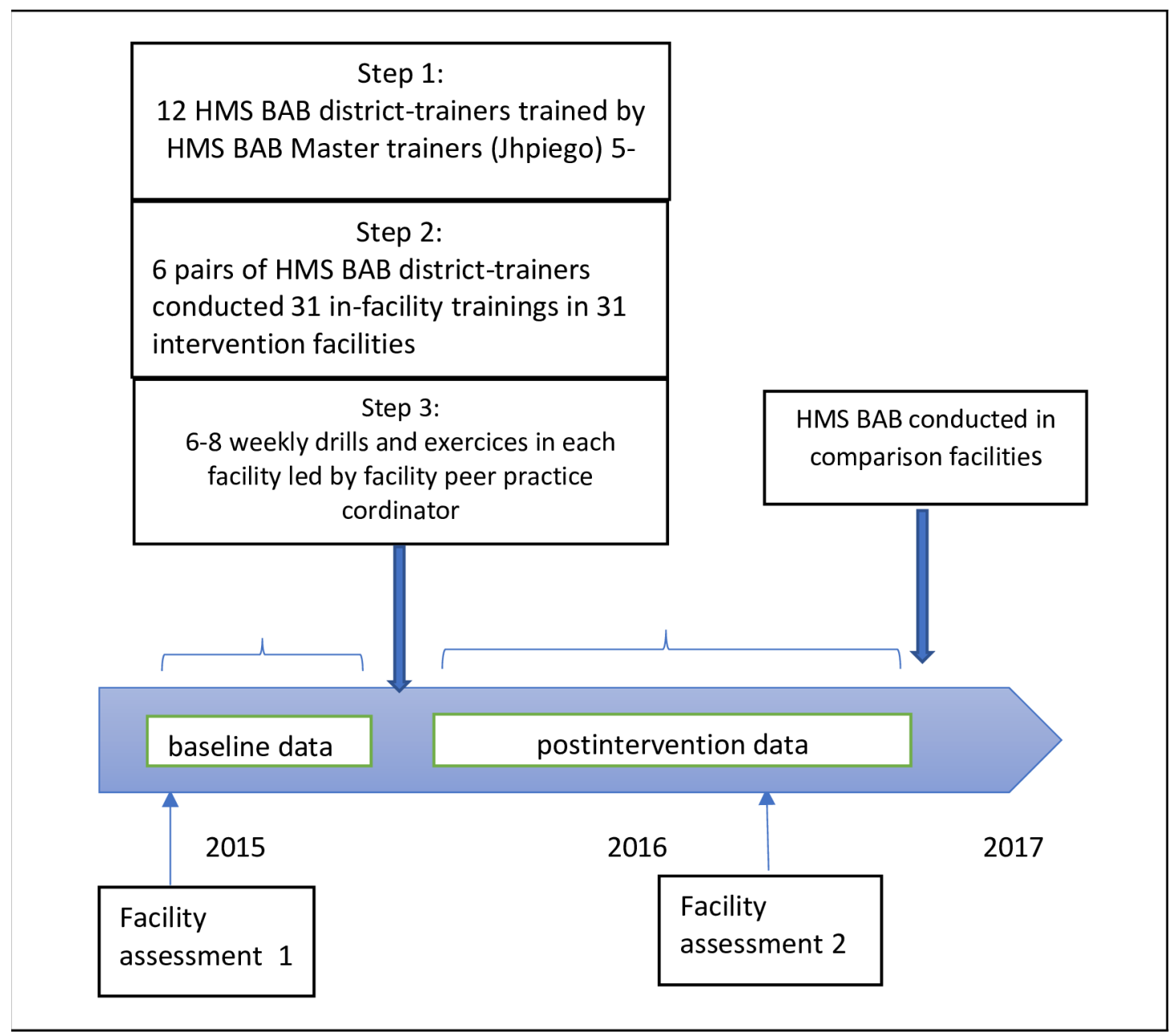

Figure 2 Timeline of the trial. HMS BAB, Helping Mothers Survive Bleeding after Birth.

advance. Providers who were off duty or scheduled to work different shifts on those dates were still requested to attend the training. When available, district reproductive health coordinators or their representatives accompanied trainers to ensure the involvement of district administrators for mentoring capacity. The district trainers made an additional supervision visit and weekly calls to peer practice coordinators to follow-up during the first 3 months after step 2 training.

All trainings used the standard HMS BAB curriculum and training materials; a set of the materials including a Mama Natalie was donated to each of the facilities.

\section{Outcomes}

We defined three primary outcome indicators: (1) all-cause near misses among all women who delivered in the facility, (2) PPH near misses among all women who delivered in the facility, and (3) PPH near misses among women who suffered PPH during facility delivery. We assessed changes in the trends of the occurrence between intervention and comparison district cluster-level estimates. ${ }^{23}$ We defined PPH near miss case fatality as a death of a mother who experienced PPH near miss. We defined secondary outcome indicators as (1) interventions provided to prevent and manage $\mathrm{PPH}$ such as:
AMTSL, oxytocin administration, manual removal of placenta, receipt of a blood transfusion, hysterectomy, and (2) changes in providers' knowledge and competencies in PPH care. The latter was assessed using a standard knowledge questionnaire as well as a checklist of observations from the simulated drills. Thus, we included a comprehensive evaluation from reaction to effects along the implementation pathway according to the Kirkpatrick model. ${ }^{29}$

\section{Sample size}

We assumed all-cause near miss and PPH near miss prevalence of 6 and 2 per 100 deliveries, respectively ${ }^{30} 31$ and aimed to observe a $25 \%$ reduction in the proportion of the baseline primary indicator at $80 \%$ power and $5 \%$ error and an intercluster factor (k) of 0.15 . The sample size was 10 clusters in each trial arm, with 3000 deliveries per cluster. ${ }^{23}$

\section{Randomisation}

Districts were enrolled after consultation with regional administrations and on fulfilment of inclusion criteria. ${ }^{14}$ We matched the districts using information from a baseline health facility survey and baseline near miss data collection which included: number of hospitals and 
health centres per district, rural/urban districts, Lake and Southern zone districts and proportion of primary outcomes reported during the baseline period. Ten matched district pairs were created, half of each pair was randomly allocated to be in either the intervention or comparison arm of the trial. A biostatistician, independent of the implementation team, randomised and allocated clusters to reduce selection bias (used STATA software V.15). The intervention did not allow for blinding of implementers or providers.

\section{Data collection}

We collected near misses from all enrolled facilities prospectively. We adapted the WHO's disease and management-based near miss tool for data collection and included background information on age, parity, induction/augmentation of labour and estimated blood loss. ${ }^{31}{ }^{32}$ We estimated the amount of PPH blood loss using visual observation and encouraged providers to employ the kanga (traditional cloth) method which uses a number of soaked kangas to estimate blood loss. ${ }^{33}$

We trained two nurse-midwives or clinicians as data collectors per facility. Training, retraining and supervision of data collectors are described in the protocol paper. ${ }^{23}$ Abstracted data were entered electronically (Lenovo tablets A3500F) and uploaded to a secure server on a weekly basis. Continuous data checks on form completion and upload status were done by the national study coordinator. ${ }^{14}$ The national study coordinator made monthly telephone calls to collect information on all facility deliveries. The coordinator also made supervision visits at first every month, then every quarter to verify the data collected against the facility registers, including looking at case notes. All data collectors were connected to a WhatsApp message group for continuous support.

We conducted two health facility assessments, one at baseline (May 2015) and one postintervention (October 2016), to understand the district health infrastructure and readiness for PPH care. We used a modified checklist from Tanzania service provision assessment tools combined with information from similar in-country studies to assess facility readiness to provide $\mathrm{PPH}$ interventions. ${ }^{14}$

In addition, all providers who attended the training completed a before-and-after assessment on knowledge and competencies on detecting and managing $\mathrm{PPH} .{ }^{19} 20$

\section{Statistical analysis}

We used STATA software V.15 for all analysis and based the analysis on 'intention to treat'. The unit of the analysis was the district. Descriptive variables obtained from individual health facility assessments were collapsed to reflect district estimates. Numerical variables were presented as medians with IQRs, and categorical variables were presented as proportions with 95\% CIs. Mann-Whitney non-parametric test and $\chi^{2}$ test were used to compare the two trial groups at baseline and postintervention periods. $P$ values less than 0.05 were considered significant.
For the effect evaluation we included 6-month baseline and 10-month postintervention assessment in all 20 districts. Interrupted time series analysis was used to estimate seven parameters for each primary and secondary outcome. ${ }^{34}$

1. The preintervention trend (slope) in the comparison districts.

2. The difference in the mean (intercept) outcome between intervention and comparison districts prior to the intervention.

3 . The difference in the slope (trend) of the outcome between intervention and comparison districts prior to the intervention.

4. The change in the level of the outcome that occurs in the period immediately following the introduction of the intervention in the comparison districts (shortterm effect).

5. The difference between preintervention and postintervention slopes of the outcome (long-term effect) in the comparison districts.

6 . The difference between intervention and comparison districts in the level of the outcome variable immediately following introduction of the intervention (short-term effect).

7. The difference between intervention and comparison districts in the slope (trend) of the outcome after initiation of the intervention compared with preintervention (long-term effect).

We used interrupted time series analysis in order to compare the trends over time in the outcomes of interest, between intervention and control clusters before and after the intervention period and, by doing so, to adjust for baseline differences between intervention and comparison groups.

\section{Ethical considerations}

The study received ethical clearance from the Senate Research and Publication Committee of Muhimbili University of Health and Allied Health Science. Written informed consent to include all facilities was gained from the district medical officers as well as the medical officers in charge of the selected facilities. Written consent was gained from all providers who were trained and were assessed during the intervention. No consent was sought from individual women as we only used facility data routinely collected in case notes and registries. All data were deidentified prior to electronic entry and storage.

\section{RESULTS}

The trial took place in all of the intervention and comparison facilities enrolled, with no facility dropping out. Two of the 200 postintervention months of data collection were missed as facilities failed to report data (one each from an intervention and comparison district); both were remote low case load facilities. We imputed the missing values by calculating the average of district-month data points lying before and after the missing data point. All 
Table 1 Facility readiness to care for women with $\mathrm{PPH}$ : staffing, case load and infrastructure by intervention and comparison districts

\begin{tabular}{|c|c|c|c|c|c|c|}
\hline \multirow[b]{2}{*}{$\begin{array}{l}\text { Readiness } \\
\text { characteristics }\end{array}$} & \multicolumn{2}{|l|}{$\begin{array}{l}\text { Baseline } \\
\text { (June 2015) }\end{array}$} & \multirow[b]{2}{*}{$P$ value } & \multicolumn{2}{|l|}{$\begin{array}{l}\text { Postintervention } \\
\text { (October 2016) }\end{array}$} & \multirow[b]{2}{*}{ P value } \\
\hline & $\begin{array}{l}\text { Intervention } \\
\text { ( } n=10 \text { districts) }\end{array}$ & $\begin{array}{l}\text { Comparison } \\
\text { ( } n=10 \text { districts) }\end{array}$ & & $\begin{array}{l}\text { Intervention } \\
\text { ( } n=10 \text { districts) }\end{array}$ & $\begin{array}{l}\text { Comparison } \\
\text { ( } n=10 \text { districts) }\end{array}$ & \\
\hline $\begin{array}{l}\text { Number of clinicians, } \\
\text { median (IQR) }\end{array}$ & $29(16-37)$ & $22(18-29)$ & $0.38^{*}$ & $26(20-45)$ & $25(17-31)$ & $0.40^{\star}$ \\
\hline $\begin{array}{l}\text { Number of nurse- } \\
\text { midwives, median } \\
\text { (IQR) }\end{array}$ & $72(35-120)$ & $61(21-103)$ & $0.29^{*}$ & $60(55-132)$ & 75 (57-122) & $0.67^{\star}$ \\
\hline $\begin{array}{l}\text { Deliveries/month, } \\
\text { median (IQR) }\end{array}$ & $292(202-411)$ & 263 (198-290) & $0.54^{*}$ & 361 (211-469) & 313 (192-405) & $0.82^{*}$ \\
\hline $\begin{array}{l}\text { Facilities with PPH } \\
\text { protocol, \% (95\% Cl) }\end{array}$ & 58 (33 to 75$)$ & 63 (14 to 55$)$ & $0.17 \dagger$ & 88 (73 to 100$)$ & 70 (47 to 92 ) & $0.37 \dagger$ \\
\hline $\begin{array}{l}\text { Facilities with } \\
\text { oxytocin in labour } \\
\text { ward, \% (95\% Cl) }\end{array}$ & 89 (77 to 100$)$ & 97 (89 to 100$)$ & $0.77 \dagger$ & 98 (93 to 100$)$ & 97 (89 to 100) & $0.68 \dagger$ \\
\hline $\begin{array}{l}\text { Facilities with blood } \\
\text { transfusion capacity, } \\
\%(95 \% \mathrm{Cl})\end{array}$ & 35 (16 to 53 ) & 32 (11 to 51$)$ & $0.67 \dagger$ & 45 (22 to 68 ) & 43 (20 to 66 ) & $0.45 \dagger$ \\
\hline $\begin{array}{l}\text { Proportion of } \\
\text { facilities with written } \\
\text { referral protocol, \% } \\
(95 \% \mathrm{Cl})\end{array}$ & 5.3 (0 to 13$)$ & 8.3 (0 to 17$)$ & $0.58 \dagger$ & 26 (8 to 43 ) & 28 (4 to 51$)$ & $0.55 \dagger$ \\
\hline $\begin{array}{l}\text { Facilities with } \\
\text { transport for referral, } \\
\%(95 \% \mathrm{Cl})\end{array}$ & 44 (28 to 59$)$ & 35 (13 to 56$)$ & $0.39 \dagger$ & 57 (37 to 75 ) & 48 (20 to 74$)$ & $0.50 \dagger$ \\
\hline
\end{tabular}

Blood transfusion capacity is defined by having five or more screened blood units in stock in the facility on the day of the survey. Transport for referral is defined as a working motorised vehicle with fuel and driver available on the day of the survey. Clinicians refer to medical officers, assistant medical officers and clinical officers. Nurse-midwives refer to enrolled (certificate) and registered (diploma and above) nurses and midwives. The number of clinicians and nurse-midwives is per district and includes all staff employed in the facilities rather than just in the labour ward. The readiness items were captured from two facility assessments conducted, one at baseline (May 2015) and one at end line (October 2016).

${ }^{*} P$ value using Mann-Whitney non-parametric test.

†T-test.

$\mathrm{PPH}$, postpartum haemorrhage.

facilities, except for two, were trained in January 2015. Two were delayed due to one facility being unreachable due to rain, and the other experiencing an acute staff shortage due to competing official work. Training was held in these two facilities in February 2015.

Table 1 shows that intervention and comparison districts had similar levels of facility readiness at baseline and postintervention surveys. Most labour wards had oxytocin available: $89 \%$ (95\% CI $89 \%$ to $100 \%)$ in intervention district and $89 \%$ (95\% CI $77 \%$ to $100 \%$ ) in comparison district, respectively. Referral readiness was insufficient in both, with less than half of the facilities having functional transport on the day of the assessment.

We trained 331 providers of different cadres working in the maternity sections: $12 \%$ were clinicians, $67 \%$ nurse-midwives, $19 \%$ medical attendants and six laboratory technicians and nursing students. Eighty per cent completed the training (268/331). Table 2 shows the mean pretraining $\mathrm{PPH}$ knowledge score of $74 \%$ which increased by $13 \%$ points after the training (95\% CI 10 to 16). Skills assessment (from simulations) showed the following gains: $49 \%$ point increase for AMTSL (95\% CI 41 to 57$), 42 \%$ point increase for recognition of retained placenta (95\% CI 32 to 50 ) and $42 \%$ point increase (95\% CI 39 to 45) for management of severe PPH.

We included 120533 facility deliveries in our data set (66 380 in intervention and 54173 in comparison districts). Facilities reported 6503 cases of all-cause near misses and 202 maternal deaths during the trial period (figure 3). We saw no clinically relevant differences in relation to age and parity of women included in the study (table 3).

We observed no significant differences in two of the primary indicators: all-cause near misses and PPH near misses among all women who delivered in a facility (table 4, figure 4). There was a significant downward trend of PPH near miss cases among women who suffered PPH in facility delivery in intervention districts compared with the comparison districts (difference-in-differences of slopes $-5.3,95 \%$ CI -7.8 to $-2.7, \mathrm{p}<0.001)$. Among 
Table 2 Knowledge and competencies (from simulations) before and after HMS BAB

\begin{tabular}{|c|c|c|c|c|c|}
\hline & Providers & $\begin{array}{l}\text { Pretraining score } \\
\text { mean/median }\end{array}$ & Providers & $\begin{array}{l}\text { Post-training score } \\
\text { mean/median }\end{array}$ & $\begin{array}{l}\text { Adjusted change } \\
\text { in score* (pre- } \\
\text { post) }\end{array}$ \\
\hline & $\mathbf{n}$ & $\%$ & $\mathbf{n}$ & $\%$ & $\%(95 \% \mathrm{Cl})$ \\
\hline Knowledge & 326 & $74 / 80$ & 301 & $87 / 93$ & 13 (10 to 16$)$ \\
\hline AMTSL simulation & 317 & $32 / 33$ & 284 & $83 / 87$ & 49 (41 to 57 ) \\
\hline $\begin{array}{l}\text { Recognition of retained } \\
\text { placenta }\end{array}$ & 281 & $47 / 50$ & 268 & $90 / 100$ & 42 (34 to 50$)$ \\
\hline PPH management & 284 & $35 / 33$ & 268 & $79 / 83$ & 44 (39 to 48$)$ \\
\hline
\end{tabular}

*Derived from a multilevel mixed methods model with fixed effects specified for the district and the hospital level, adjusted for the health work cadre. A total of 268 out of 331 providers included in the training completed the HMS BAB training. Providers left the training to attend emergencies or other duties.

AMTSL, active management of the third stage of labour; HMS BAB, Helping Mothers Survive Bleeding after Birth; PPH, postpartum haemorrhage.

secondary indicators, the interrupted time series analysis indicated that more women who suffered PPH were treated with intravenous oxytocin (difference-in-differences of slopes 5.2, 95\% CI 1.4 to 8.9) $(\mathrm{p}<0.01)$ and fewer women received a blood transfusion (difference-in-differences of slopes $-8.0,95 \% \mathrm{CI}-12.6$ to $3.4, \mathrm{p}<0.01$ ) in the intervention compared with comparison districts (table 4, figure 5). Differences in the intercept and short-term and long-term trends are shown in the online supplementary table. We observed no difference between intervention and comparison districts for other secondary indicators which included: discharge with haemoglobin $<70 \mathrm{~g} / \mathrm{L}$, removal of retained placenta or women treated with hysterectomy.

A total of $81.5 \%$ of women who suffered PPH during facility delivery were reported to have had received AMTSL and $92.4 \%$ received intravenous oxytocin during baseline data assessment (comparison group).

A similar table showing differences in indicators at baseline, short-term and long-term effects between intervention and comparison districts is shown in the online supplementary table.

We reanalysed data using a modified near miss definition to include cases with two units of blood transfused (compared with one unit) as advised by the data advisory board. Our results were weakened and lost significance though the direction of change remained constant (online supplementary figure 1).

\section{DISCUSSION}

\section{Main findings}

We observed a strong protective short-term and longterm effects of the training on PPH near misses among women who suffered PPH during health facility delivery in intervention compared with comparison districts. Importantly, we also saw significant reductions in case fatality in PPH cases in the intervention compared with the comparison districts, although a small effect. There was no evidence of any effect on all-cause near misses,
PPH near misses in all deliveries or occurrence of PPH in all deliveries.

Our study was conducted in typical rural districts in two distinct zones in Tanzania, which makes the study findings applicable to other areas of Tanzania. Our findings imply that once a PPH occurred, a lower proportion of women deteriorated enough to be classified as PPH near misses. We hypothesise, taking our theory of change into consideration, first, that recognition of severe bleeding improved as the training emphasised blood loss estimation, enhanced early PPH detection and increased vigilance and diagnosis of cases. This was also reported in other competence-based training studies. ${ }^{35-38}$ Second, our post-training assessment indicated that health providers were more competent to diagnose and manage PPH following the frequent practised drills. This has also been shown by other studies. ${ }^{19} 21223539$ As expected, once PPH was diagnosed, health providers could intervene early and prevent women deteriorating to a more severe condition. Third, the training included all providers working in the maternity sections. This inclusive, holistic approach of the training may have promoted continued learning and teamwork within the facility which further enforced the first two effects.

We observed high implementation levels for AMTSL as well as management of PPH with oxytocin, which is encouraging. While we cannot exclude the possibility of a reporting bias (as our estimates are based on health providers' reports), estimates compare well with those from an observational study in Tanzania that also reported proportions of AMTSL implementation above $90 \% .{ }^{35}$ Our pretraining assessment indicated a reasonable baseline knowledge of prevention and treatment of PPH while skills scores were lower. Various emergency obstetric care training courses have been implemented in the country and health workers are aware that AMTSL ought to be done. ${ }^{13} 3640$ The reported high implementation levels of AMTSL could also be the reason that we could not see a reduction of $\mathrm{PPH}$ in this trial as other studies evaluating HMS BAB did..$^{35}$ 


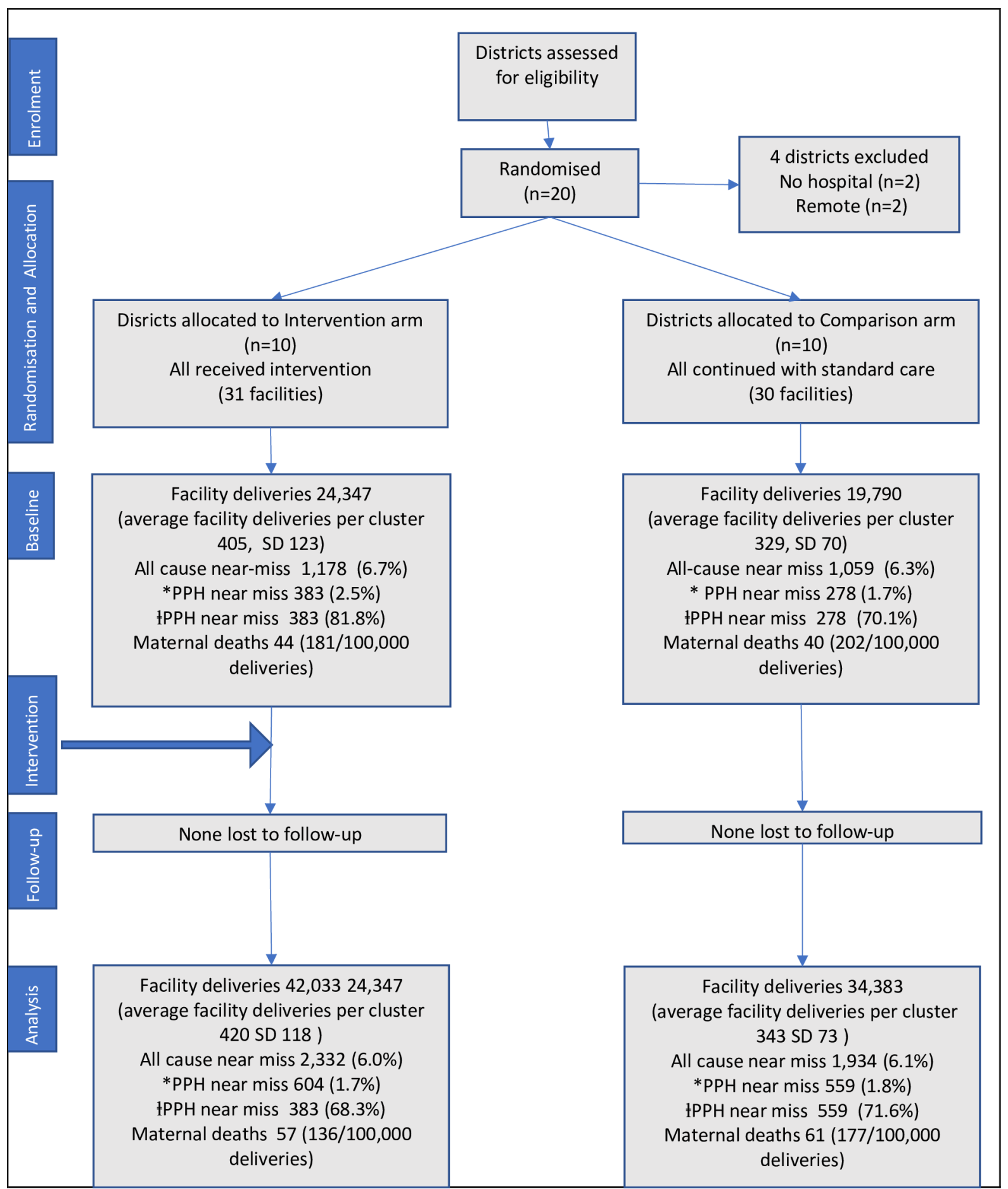

Figure 3 Trial flow chart. PPH Postpartum hemorrhage

Similar to this trial, a different simulated training in Mexico, Programa de Rescate Obstétrico y Neonatal Tratamiento Óptimo y Oportuno, reported improved clinical practices but no reduction in obstetric haemorrhage cases $^{41}$ — probably also because AMTSL was already well implemented.

We found a significant reduction in the proportion of women receiving a blood transfusion following PPH in intervention districts, similar to those reported elsewhere. ${ }^{37}$ We observed an improved availability of blood in both trial groups during the trial period, following a change in blood collection and distribution services from zonal to district level in Tanzania. This supports the hypothesis that early recognition and administration of intravenous oxytocin may have reduced the severity of the PPH. However, it is important to note that most women only received a single unit of blood transfusion (median 1.5, IQR 1-2, data not shown) as there is large demand on blood supplies for other conditions such as pregnant women with anaemia $(11 \%$ of reproductive 
Table 3 Sociodemographic characteristics of women who were included into the study

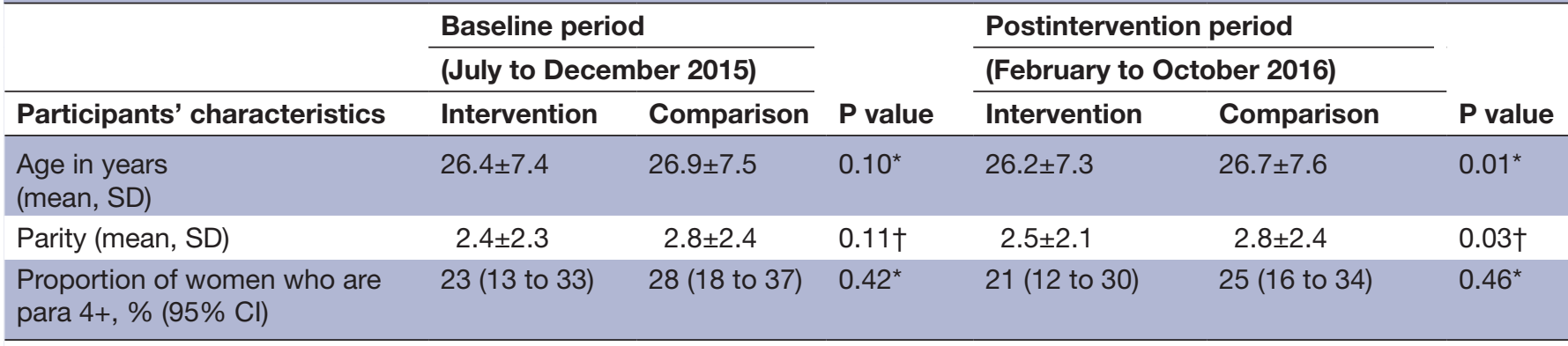

${ }^{\star}$ T-test.

†Wilcoxon rank-sum test.

aged women have moderate anaemia and 1\%-2\% have severe anaemia) ${ }^{24}$ as well as paediatric conditions or HIV/ AIDS. The very restricted use of blood transfusion is also supported by our finding that almost $20 \%$ of women who had their haemoglobin checked were discharged with a level below $70 \mathrm{~g} / \mathrm{L}$ and thus with a suboptimal treatment.

While we saw an encouraging increase in the use of prompt basic management intervention, we observed very few cases where additional life-saving interventions such as intrauterine balloon tamponade, B-lynch sutures, drugs like misoprostol or tranexamic acid were used. More advanced management and technology needs to be implemented, however, consistently to reduce morbidity and mortality further. Since the implementation of this HMS BAB training a second module was developed and tested which includes additional elements such as manual removal of the placenta, cervical laceration repair and advanced medical management including use of tranexamic acid (the module is called 'HMS BAB complete'). ${ }^{28}$

\section{Strength and limitations}

The strength of this trial is that we included 61 facilities and over 120000 deliveries from both the northern and southern parts of the country. While we aimed to include 'typical' districts, the structure however differed slightly with some districts having more hospitals or facilities offering caesarean section. This created imbalances within the trial population including the total number of deliveries. However, we adjusted in the interrupted time series analysis for these baseline imbalances. We present our trial as an effectiveness trial which was undertaken in typical conditions with resource constraints. Our two facility assessments indicated severe shortages in drugs, supplies and the referral system; there was no evidence of difference in the overall resource level between the two trial arms. Three health centres were upgraded to become comprehensive emergency obstetric care facilities and one health centre was upgraded into a district hospital as part of the national road map to improve maternal and newborn health. Consequently, the number of deliveries in these facilities increased. We also report on improvements: availability of blood and the number of facilities equipped to provide emergency obstetric care increased during the trial, including having a written referral plan. These concurrent improvements were part of the nationwide efforts to improve maternal and newborn health and might have biased our results, probably towards weakened estimates of effect.

Our evaluation was based on the theory of change proposed by Kirkpatrick. We report on (1) improved knowledge and skills (increased simulated skills scores, table 2) and increased availability of protocols (table 1); (2) improved application of curative interventions (intravenous oxytocin and reduced need for blood transfusion, table 4); and (3) reduced $\mathrm{PPH}$ near miss rate, reduced case fatality rate and thus mortality and morbidity outcomes (table 4). ${ }^{29}$ This is different from most other evaluations which only reported a positive effect on knowledge and skills but were not designed to indicate improved practices and any effects on mortality and morbidity outcomes. ${ }^{13}$

We used an accepted methodology to define morbidity using the WHO near miss definition. Our near miss estimate of 6.8 near misses per 100 deliveries compares well with other estimates. ${ }^{31} 32$ 42-44 Tunçalp and Souza in a systematic review reported a prevalence of $0.6 \%-14.9 \%$ using disease-specific criteria and $3.15 \%$ using a combination of disease and management-specific criteria in African countries. ${ }^{45}$ We included as near misses all those cases with one or more blood transfusions as a management criterion as proposed by Nelissen et al (2013) ${ }^{31}$ and reanalysed using two blood transfusions. We believe that any blood transfusion is a marker of severity in districts reporting shortage of supply of blood.

We relied on trained maternity staff to collect data which is in contrast to most other near miss studies who employed research nurses. This might have affected the completeness and reliability of the data, and could have resulted in reporting bias. Two other studies in the region which assessed maternal near miss prevalence and quality of care have used health facility staff involved in maternity care for data collection with study staff supervision, and reported acceptable levels of data completion and accuracy. ${ }^{4647}$ We performed regular supervision visits to verify the reported data against registers and case notes. Also, regular mobile calls by our study team aimed to support 


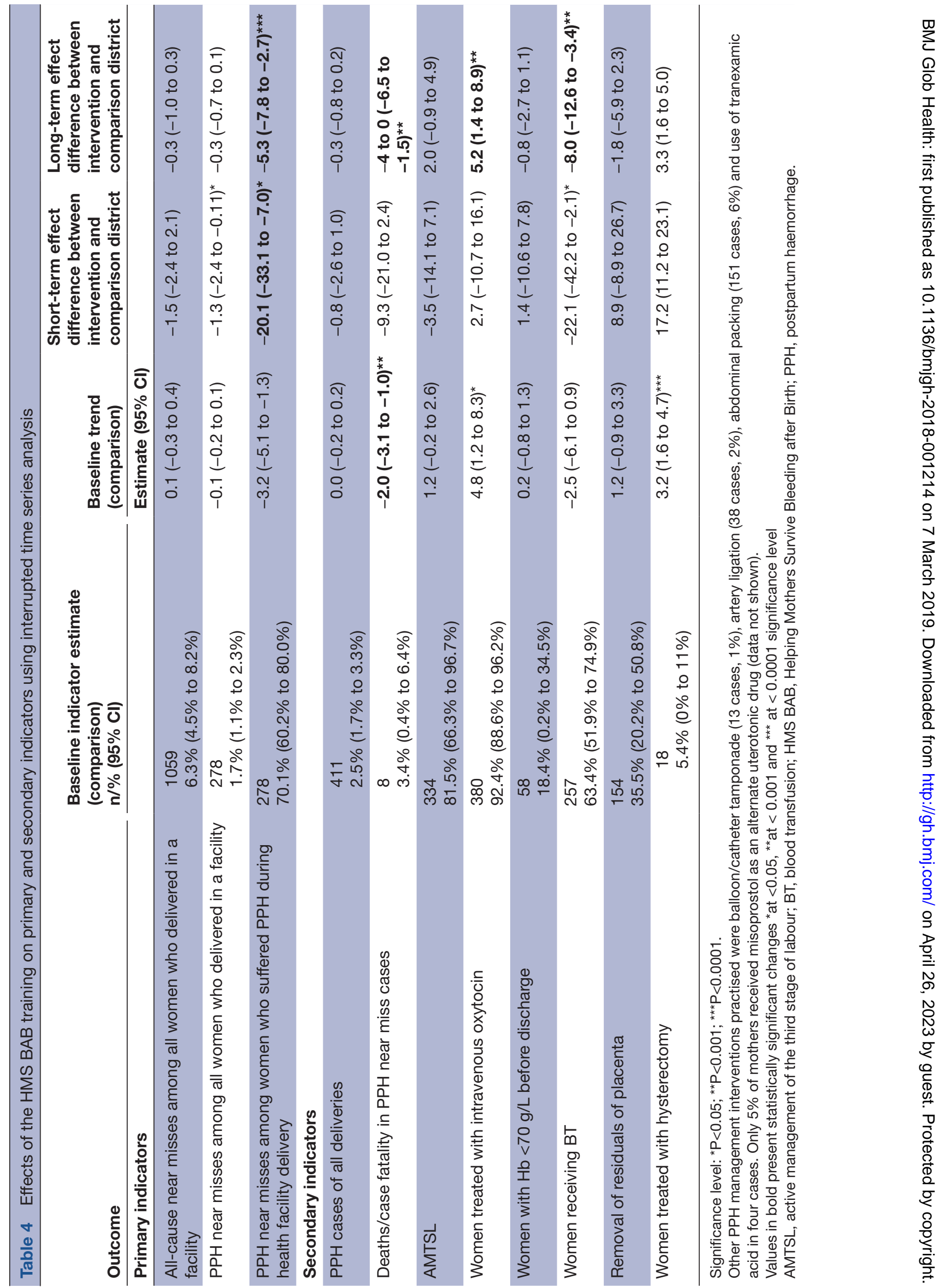



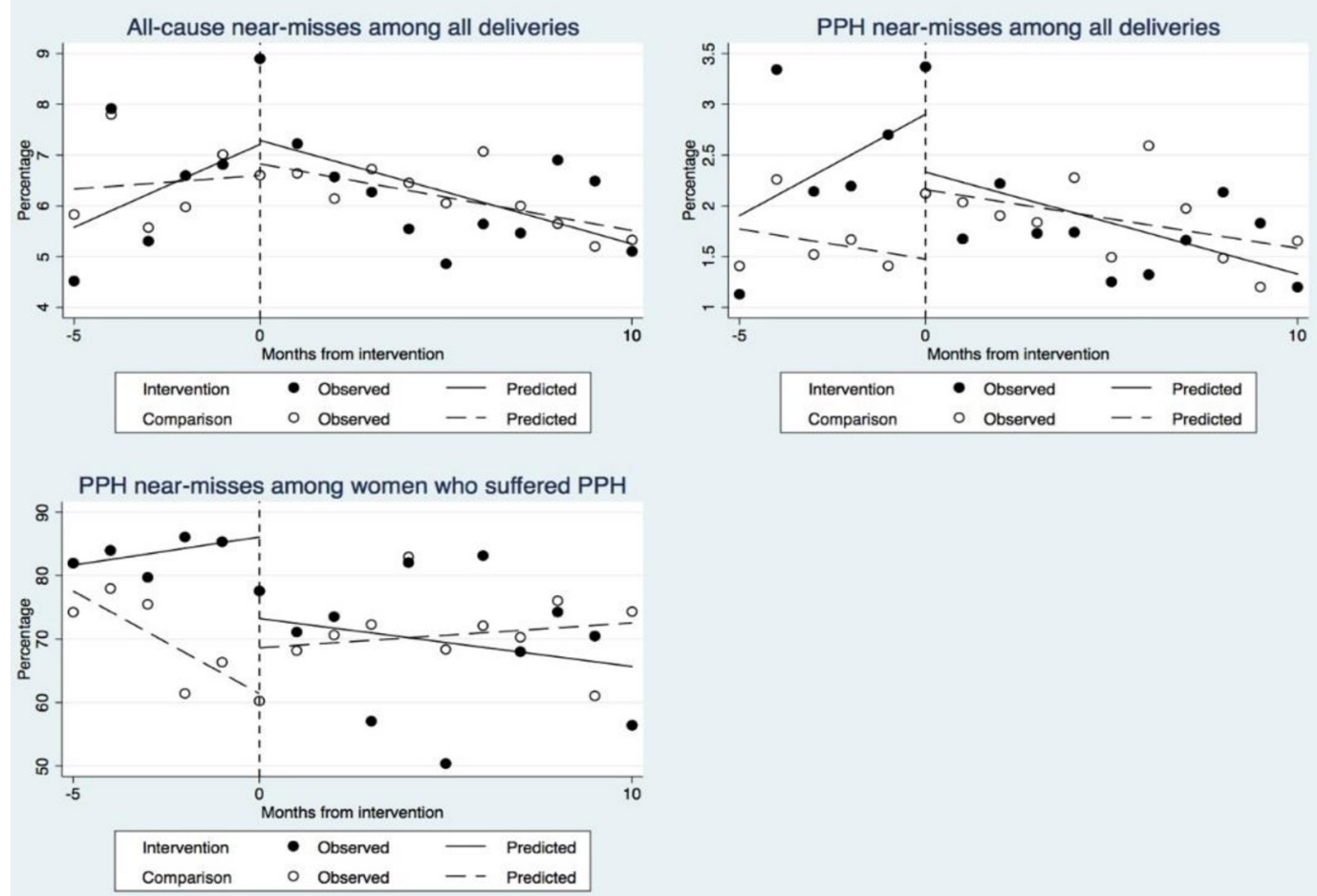

Figure 4 Change of trends in the three primary outcome indicators from baseline to postintervention. PPH, postpartum haemorrhage.
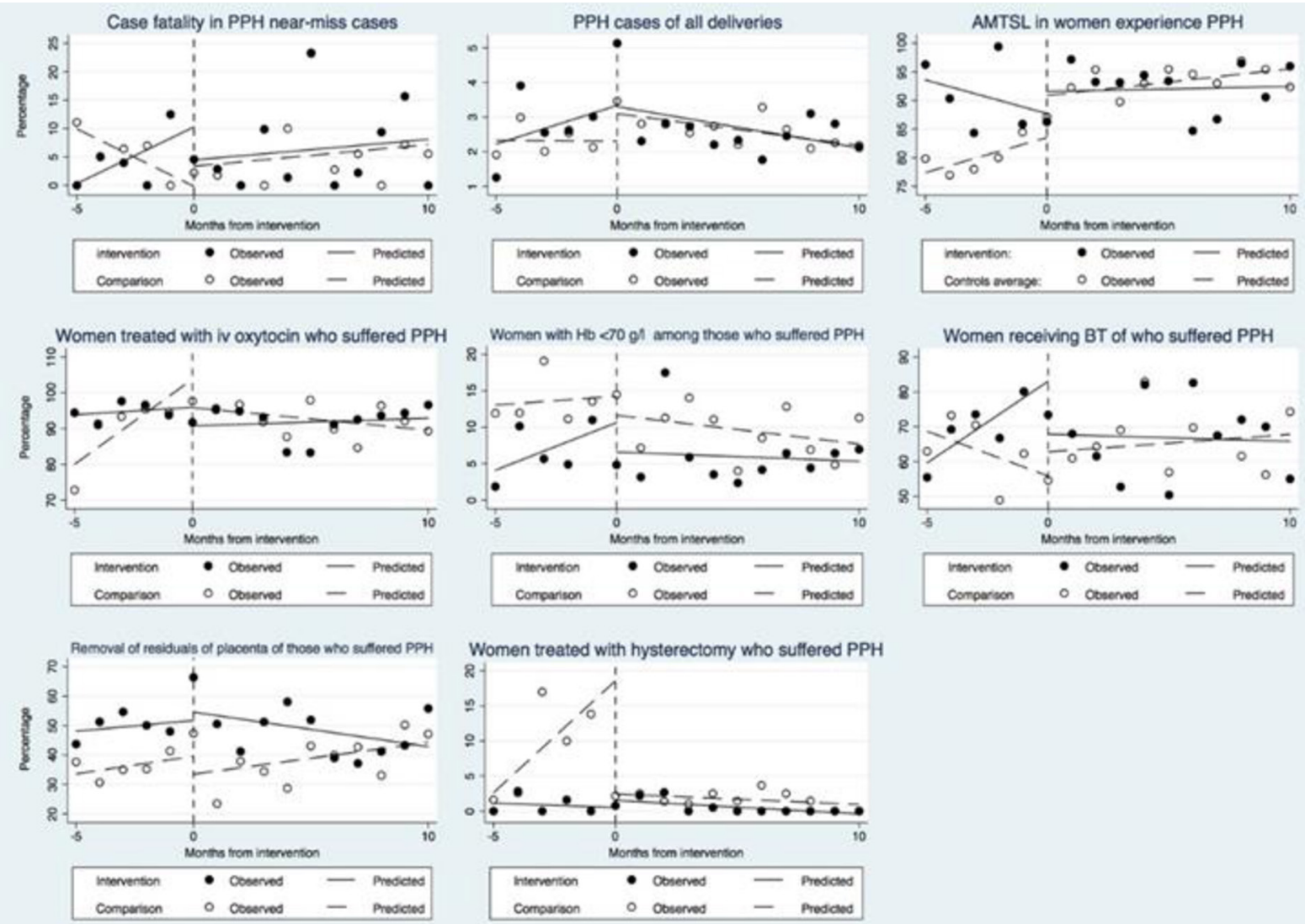

Figure 5 Change of trends of the secondary outcome indicators from baseline to postintervention. AMTSL, active management of the third stage of labour; BT, blood transfusion; PPH, postpartum haemorrhage. 
the completeness and accuracy of reporting. In addition, a quality assurance exercise carried out in a third of the facilities showed only small differences between captured cases and registers/case notes. We report on documented practices and outcomes, thus relying on appropriately filled registers and case notes which is itself a challenge due to potential under-reporting as health workers prioritise patient care above documentation if the case load is high. However, as our estimates of $\mathrm{PPH}$, implementation of AMTSL, intravenous oxytocin and other estimates compare well with a recently published study using observation measures, ${ }^{48}$ we believe that the approach we used was acceptable. Moreover, data were consistent during baseline and postintervention leading us to assume that no differential bias was introduced.

PPH was diagnosed by using the kanga method-saturation of the standard-sized local cloth. ${ }^{33}$ When conceptualising the trial we explored other options such as the blood mat, but the purchase price was beyond our financial capacity-a problem also faced by Nelissen et al..$^{35}$ This could have led to an under-reporting of PPH cases. However, we believe no differential under-reporting was introduced as intervention and comparison facilities both faced this challenge similarly.

\section{CONCLUSION}

Our large cluster-randomised trial conducted in typical rural districts in Tanzania indicated a significant, although small reduction of PPH near misses after the implementation of the 1-day in-facility HMS BAB training. We saw a positive effect of the training along the implementation line from knowledge and skills to selected implementation practices to reduction of PPH-related near misses and mortality. However, as case fatality is still high, training might need to include additional interventions such as use of tranexamic acid and intrauterine balloon placement. While training is clearly important, it is insufficient on its own to bring the much-needed reduction in $\mathrm{PPH}$ near misses and mortality.

\section{Author affiliations \\ ${ }^{1}$ Department of Public Health Sciences, Karolinska Institutet, Solna, Sweden ${ }^{2}$ Department of Obstetrics and Gynaecology, Muhimbili University of Health and Allied Sciences, Dar es Salaam, Tanzania \\ ${ }^{3}$ International Federation of Gynecology and Obstetrics (FIGO), London, UK ${ }^{4}$ School of Nursing, Muhimbili University of Health and Allied Sciences, Dar es Salaam, Tanzania \\ ${ }^{5}$ Department of Disease Control, London School of Hygiene and Tropical Medicine, London, UK}

Acknowledgements We thank the Tanzania's Ministry of Health, Community Development, Gender, Elderly and Children (MoHCDGEC) for guidance and support, regional and district health management teams and administrators and health workers, women and their families for participating in the study. The trial was done as a collaboration between two local professional associations: The Association of Gynaecologists and Obstetricians of Tanzania (AGOTA) and Tanzania Midwifery Association (TAMA). We thank the HMS BAB study team for Tanzania and Uganda: Hilda Kwezi, Lucy Joyce Atim, Shirima Kizito, Dinah Amongin and Nester Moyo of International Confederation of Midwives for all the support and work that went into implementing the trial. Our sincere appreciation to the scientific advisory board members, Professor William Stones, MD, FRCOG, Professor Birgitta Essén, MD,
PhD, Associate Professor Hege Ersdal, MD, PhD, and Cherrie Evans, DrPH, CNM, for their oversight and guidance.

Contributors $\mathrm{CH}$ and FAA conceptualised the study in Tanzania with support from $\mathrm{AP}$, and JM. GM, FAA, AH and $\mathrm{CH}$ performed the statistical analysis. FAA, CH, AP and SL were involved into the operationalisation of the study and the data collection support. FAA and $\mathrm{CH}$ wrote the first draft of this protocol. All authors contributed to the manuscript development and commented on several drafts of the manuscript. All authors read and approved the final manuscript.

Funding This study was funded by Laerdal Foundation for Acute Medicine (40070).

\section{Competing interests None declared.}

Patient consent for publication Not required.

Ethics approval Senate Research and Publication Committee of Muhimbili University of Health and Allied Health Science

Provenance and peer review Not commissioned; externally peer reviewed.

Data sharing statement Additional data will be archived on the Dyrad Digital Repository.

Open access This is an open access article distributed in accordance with the Creative Commons Attribution Non Commercial (CC BY-NC 4.0) license, which permits others to distribute, remix, adapt, build upon this work non-commercially, and license their derivative works on different terms, provided the original work is properly cited, appropriate credit is given, any changes made indicated, and the use is non-commercial. See: http://creativecommons.org/licenses/by-nc/4.0/.

\section{REFERENCES}

1. Alkema L, Chou D, Hogan D, et al. Global, regional, and national levels and trends in maternal mortality between 1990 and 2015, with scenario-based projections to 2030: a systematic analysis by the UN maternal mortality estimation Inter-Agency group. The Lancet 2016;387:462-74.

2. Afnan-Holmes H, Magoma M, John T, et al. Tanzania's countdown to 2015: an analysis of two decades of progress and gaps for reproductive, maternal, newborn, and child health, to inform priorities for post-2015. Lancet Glob Health 2015;3:e396-409.

3. Adegoke AA, van den Broek N. Skilled birth attendance-lessons learnt. BJOG 2009;116(Suppl 1):33-40.

4. Montagu D, Sudhinaraset M, Diamond-Smith N, et al. Where women go to deliver: understanding the changing landscape of childbirth in Africa and Asia. Health Policy Plan 2017;32:1146-52.

5. Campbell OMR, Calvert C, Testa A, et al. The scale, scope, coverage, and capability of childbirth care. The Lancet 2016;388:2193-208.

6. Miller S, Abalos E, Chamillard M, et al. Beyond too little, too late and too much, too soon: a pathway towards evidence-based, respectful maternity care worldwide. The Lancet 2016;388:2176-92.

7. Chaturvedi S, Upadhyay S, De Costa A. Competence of birth attendants at providing emergency obstetric care under India's JSY conditional cash transfer program for institutional delivery: an assessment using case vignettes in Madhya Pradesh Province. BMC Pregnancy Childbirth 2014;14

8. Ariff S, Soofi SB, Sadiq K, et al. Evaluation of health workforce competence in maternal and neonatal issues in public health sector of Pakistan: an assessment of their training needs. BMC Health Serv Res 2010;10.

9. Adegoke A, Utz B, Msuya SE, et al. Skilled birth attendants: who is who? A descriptive study of definitions and roles from nine sub Saharan African countries. PLoS One 2012;7:e40220.

10. Ijadunola KT, ljadunola MY, Esimai OA, et al. New paradigm old thinking: the case for emergency obstetric care in the prevention of maternal mortality in Nigeria. BMC Womens Health 2010;10.

11. Say L, Chou D, Gemmill A, et al. Global causes of maternal death: a WHO systematic analysis. Lancet Glob Health 2014;2:e323-33.

12. Bartlett L, Cantor D, Lynam $P$, et al. Facility-based active management of the third stage of labour: assessment of quality in six countries in sub-Saharan Africa. Bull World Health Organ 2015;93:759-67.

13. Ameh CA, van den Broek N. Making it happen: training health-care providers in emergency obstetric and newborn care. Best Pract Res Clin Obstet Gynaecol 2015;29:1077-91.

14. Hanson C, Ronsmans C, Penfold S, et al. Health system support for childbirth care in southern Tanzania: results from a health facility census. BMC Res Notes 2013;6. 
15. Blank A, Prytherch H, Kaltschmidt J, et al. "Quality of prenatal and maternal care: bridging the know-do gap" (QUALMAT study): an electronic clinical decision support system for rural Sub-Saharan Africa. BMC Med Inform Decis Mak 2013;13.

16. Calvert KL, McGurgan PM, Debenham EM, et al. Emergency obstetric simulation training: how do we know where we are going, if we don't know where we have been? Aust N Z J Obstet Gynaecol 2013;53:509-16.

17. van Lonkhuijzen L, Dijkman A, van Roosmalen J, et al. A systematic review of the effectiveness of training in emergency obstetric care in low-resource environments. BJOG 2010;117:777-87.

18. Health LG. MamaBirthie Birthing Simulator \& Skills Trainer: Laerdal Medical 2001-2018. Available: http://laerdalglobalhealth.com/ products/mamabirthie [Accessed 13 Jun 2018].

19. Nelissen $\mathrm{E}$, Ersdal $\mathrm{H}$, Østergaard D, et al. Helping mothers survive bleeding after birth: an evaluation of simulation-based training in a low-resource setting. Acta Obstet Gynecol Scand 2014;93:287-95.

20. Evans $\mathrm{CL}$, Johnson $\mathrm{P}$, Bazant $\mathrm{E}$, et al. Competency-based training "Helping Mothers Survive: Bleeding after Birth" for providers from central and remote facilities in three countries. Int $J$ Gynaecol Obstet 2014;126:286-90.

21. Nathan LM, Patauli D, Nsabimana D, et al. Retention of skills 2 years after completion of a postpartum hemorrhage simulation training program in rural Rwanda. International Journal of Gynecology \& Obstetrics 2016;134:350-3.

22. Ersdal HL, Singhal N, Msemo G, et al. Successful implementation of helping babies survive and helping mothers survive programsAn Utstein formula for newborn and maternal survival. PLOS One $2017 ; 12$.

23. Hanson C, Pembe AB, Alwy F, et al. Evaluating the effect of the helping mothers survive bleeding after birth (HMS BAb) training in Tanzania and Uganda: study protocol for a randomised controlled trial. Trials 2017;18.

24. Ministry of Health CD, Gender, Elderly and Children (MoHCDGEC) [Tanzania Mainland], Ministry of Health (MoH) [Zanzibar], National Bureau of Statistics (NBS), Office of the Chief Government Statistician (OCGS), and ICF. Tanzania demographic and Health survey and malaria indicator survey. Dar es Salaam, Tanzania, and Rockville, Maryland, USA: MoHCDGEC, MoH, NBS, OCGS, and ICF 2016.

25. Ministry of Health CD, Gender, Elderly and Children (MoHCDGEC). The National road map strategic plan to improve maternal, newborn, child and adolescent health in Tanzania 2016-2020. Dar es salaam, Tanzania, 2016.

26. Ueno E, Adegoke AA, Masenga G, et al. Skilled birth attendants in Tanzania: a descriptive study of cadres and emergency obstetric care signal functions performed. Matern Child Health $J$ 2015;19:155-69.

27. Solnes Miltenburg A, Kiritta RF, Meguid T, et al. Quality of care during childbirth in Tanzania: identification of areas that need improvement. Reprod Health 2018;15.

28. Helping mothers survive bleeding fter birth complete JHPIEGO, 2018. Available: https://hms.jhpiego.org/bleeding-after-birthcomplete [Accessed 28 Nov 2018].

29. Kirkpatrick D. Great Ideas Revisited. Techniques for Evaluating Training Programs. Revisiting Kirkpatrick's Four-Level Model. In: Training and development, 1996: 54-9.

30. Kaye DK, Kakaire O, Osinde MO. Systematic review of the magnitude and case fatality ratio for severe maternal morbidity in sub-Saharan Africa between 1995 and 2010. BMC Pregnancy Childbirth 2011;11.
31. Nelissen E, Mduma E, Broerse J, et al. Applicability of the WHO maternal near miss criteria in a low-resource setting. PLoS One 2013;8.

32. World Health Organization. Evaluating the quality of care for severe pregnancy complications: the WHO nearmiss approach for maternal health, 2011. Available: http://whqlibdoc.who.int/publications/2011/ 9789241502221_eng.pdf

33. Prata N, Mbaruku G, Campbell M. Using the kanga to measure postpartum blood loss. Int J Gynaecol Obstet 2005;89:49-50.

34. Linden A. Conducting interrupted time-series analysis for single- and multiple-group comparisons. Stata J 2015;15:480-500.

35. Nelissen E, Ersdal H, Mduma E, et al. Clinical performance and patient outcome after simulation-based training in prevention and management of postpartum haemorrhage: an educational intervention study in a low-resource setting. BMC Pregnancy Childbirth 2017;17.

36. Sorensen BL, Rasch V, Massawe S, et al. Advanced life support in obstetrics (also) and post-partum hemorrhage: a prospective intervention study in Tanzania. Acta Obstet Gynecol Scand 2011;90:609-14.

37. Egenberg S, Masenga G, Bru LE, et al. Impact of multi-professional, scenario-based training on postpartum hemorrhage in Tanzania: a quasi-experimental, pre- vs. post-intervention study. BMC Pregnancy Childbirth 2017;17.

38. Egenberg S, Karlsen B, Massay D, et al. "No patient should die of PPH just for the lack of training!" Experiences from multiprofessional simulation training on postpartum hemorrhage in northern Tanzania: a qualitative study. BMC Med Educ 2017;17.

39. Nelissen E, Ersdal H, Mduma E, et al. Helping mothers survive bleeding after birth: retention of knowledge, skills, and confidence nine months after obstetric simulation-based training. $B M C$ Pregnancy Childbirth 2015;15.

40. Ersdal HL, Singhal N, Msemo G, et al. Successful implementation of helping babies survive and helping mothers survive programsAn Utstein formula for newborn and maternal survival. PLoS One 2017;12:e0178073.

41. Walker DM, Cohen SR, Fritz J, et al. Impact evaluation of PRONTO Mexico: a simulation-based program in obstetric and neonatal emergencies and team training. Simul Healthc 2016;11:1-9.

42. Witteveen $\mathrm{T}$, Bezstarosti $\mathrm{H}$, de Koning $\mathrm{I}$, et al. Validating the WHO maternal near miss tool: comparing high- and low-resource settings. BMC Pregnancy Childbirth 2017;17.

43. Tunçalp O, Hindin MJ, Souza JP, et al. The prevalence of maternal near miss: a systematic review. BJOG 2012;119:653-61.

44. Maswime S, Buchmann E. A systematic review of maternal near miss and mortality due to postpartum hemorrhage. Int $J$ Gynecol Obstet 2017;137:1-7.

45. Tunçalp Ö, Souza JP. Maternal near-miss audits to improve quality of care. BJOG: Int J Obstet Gy 2014;121:102-4.

46. Sayinzoga F, Bijlmakers L, van der Velden K, et al. Severe maternal outcomes and quality of care at district hospitals in Rwanda- a multicentre prospective case-control study. BMC Pregnancy Childbirth 2017;17.

47. Herklots T, van Acht L, Meguid T, et al. Severe maternal morbidity in Zanzibar's referral hospital: measuring the impact of in-hospital care. PLoS One 2017;12.

48. Nelissen EJT, Mduma E, Ersdal HL, et al. Maternal near miss and mortality in a rural referral hospital in northern Tanzania: a crosssectional study. BMC Pregnancy Childbirth 2013;13. 